\title{
Imaging of pneumonia: trends and algorithms
}

\author{
T. Franquet
}

\begin{abstract}
Imaging of pneumonia: trends and algorithms. T. Franquet. (C) ERS Journals Ltd 2001. ABSTRACT: Pneumonia is one of the major infectious diseases responsible for significant morbidity and mortality throughout the world. Imaging plays a crucial role in the detection and management of patients with pneumonia.

This review article discusses the different imaging methods used in the diagnosis and management of suspected pulmonary infections. The imaging examination should always begin with conventional radiography. When the results of routine radiography are inconclusive, computed tomography is mandatory. A combination of pattern recognition with knowledge of the clinical setting is the best approach to the pulmonary infectious processes.

A specific pattern of involvement can suggest a likely diagnosis in many instances. In acquired immune deficiency syndrome patients, diffuse ground-glass and interstitial infiltrates are most commonly present in Pneumocystis carinii pneumonia whereas in the nonimmunosuppressed patients, a segmental lobar infiltrate is suggestive of a bacterial pneumonia. Round pneumonia is most often encountered in children than adults and is most often caused by Streptococcus pneumoniae. Different combinations of parenchymal and pleural abnormalities may be suggestive for additional diagnoses.

When an infectious pulmonary process is suspected, knowledge of the varied radiographic manifestations will narrow the differential diagnosis, helping to direct additional diagnostic measures, and serving as an ideal tool for follow-up examinations. Eur Respir J 2001; 18: 196-208.
\end{abstract}

\author{
Dept of Radiology, Section of Thoracic \\ Imaging, Hospital de Sant Pau, Uni- \\ versidad Autónoma de Barcelona, San \\ Antonio, Barcelona, Spain.
}

Correspondence: T. Franquet, Dept of Radiology, Section of Thoracic Imaging, Hospital de Sant Pau, Universidad Autónoma de Barcelona, San Antonio $\mathrm{M}^{\mathrm{a}}$ Claret 167, 08025 Barcelona, Spain. Fax: 932919282

Keywords: Chest radiograph

diagnosis

G-thorax

pneumonia

respiratory infections

Received: October 242000

Accepted after revision February 9 2001
Despite advances in diagnosis and treatment, pulmonary infections are a major cause of morbidity and mortality in adult patients. Pneumonia is the sixth most common cause of death in the USA and more than 6 million cases of bacterial pneumonia occur each year in the immunocompetent population [1]. The spectrum of organisms known to cause respiratory infections is broad and constantly increasing as new pathogens are identified and the host immune response is altered by medications or other diseases or responses. In the USA, it has been estimated that there are 1.1 million cases of community-acquired pneumonia (CAP) requiring hospitalization each year, at an estimated cost of 8 billion dollars [1]. Nosocomial pneumonia (NP) is the most important hospitalacquired infection because it is associated with the highest mortality rate of nosocomial infections that contribute causally to death [2]. Moreover, since the beginning of the acquired immune deficiency syndrome (AIDS) epidemic, lungs represent an increasing source of infections. In addition to direct patient care costs, pneumonia is responsible for over 50 million days of restricted activity from work and is the sixth leading cause of death in the USA with a mortality rate of 13.4 per $100,000[3,4]$.

\section{Changing trends in pulmonary infections}

Diagnosis of pneumonia calls for a combination of clinical awareness, appropriate microbiological tests, and radiographical studies. Plain chest radiography is an inexpensive test that can rapidly demonstrate the presence of pulmonary abnormalities. It represents an important initial examination in all patients suspected of having a pulmonary infection. In most cases the plain film findings may be diagnostic of pneumonia and may eliminate the need for additional radiographic procedures.

The clinician evaluating the patient with a known or suspected diagnosis of pulmonary infection faces a diagnostic challenge because of the majority of

Previous articles in this series: No. 1: Ghaye B, Dondelinger RF. Imaging guided thoracic interventions. Eur Respir J 2001; 17: 507-528. No. 2: Vansteenkiste JF, Stroobants SG. The role of positron emission tomography with ${ }^{18}$ F-fluoro-2-deoxy-D-glucose in respiratory oncology. Eur Respir J 2001; 17: 802-820. No. 3: Kauczor HU, Chen XJ, van Beek EJR, Schreiber WG. Pulmonary ventilation imaged by magnetic resonance: at the doorstep of clinical application. Eur Respir J 2001; 17: 1008-1023. No. 4: Hansell DM. Small airways diseases: detection and insights with computed tomography. Eur Respir $J$ 2001; 17: 1294-1313. 
processes presenting with similar signs and symptoms, and the radiographic findings of pneumonia do not provide a specific aetiological diagnosis. Furthermore, radiographic manifestations of a given infectious process may be variable depending on the immunological status of the patient as well as by pre- or coexisting lung disease. The number of immunocompromised patients has dramatically increased because of three phenomena: the AIDS epidemic, advances in cancer chemotherapy, and expanding organ transplantation. At the onset of the AIDS epidemic, in the early and mid $1980 \mathrm{~s}$, there was $50-80 \%$ mortality for each episode of Pneumocystis carinii pneumonia (PCP). Since routine prophylaxis was instituted in 1989, a declining incidence of PCP in the AIDS population was demonstrated $[5,6]$. In addition to lesser incidence, there was also a declining mortality $(15 \%)$ in mild-to-moderate cases [7]. Therefore, other infections including bacterial pneumonia, fungal infection, cytomegalovirus (CMV), Mycobacterium avium complex (MAC), and tuberculosis remain a significant cause of morbidity and mortality in these patients [5-7]. Radiologists must not only document the location and extent of pneumonia but also assess the evolution and course of pneumonia and detect any complication of the disease.

\section{Integrating clinical and imaging findings}

The most useful imaging modalities available for the evaluation of the patient with known or suspected pulmonary infection are chest radiography and computed tomography (CT). Imaging examinations should always be interpreted with a knowledge of how symptomatic the patient is, the degree of dyspnoea, the level of impairment of the carbon monoxide diffusing capacity of the lung ( $D \mathrm{~L}, \mathrm{CO})$, the CD4+ cell count, the presence of fever or leukocytosis, if there is a cough and whether the cough is productive, and the chronicity of symptoms [8]. Knowledge of whether the patient has developed a CAP or NP, as well as knowledge of the immune status of the patient, can be powerful tools in arriving at a shortlist of possible causative organisms [8, 9]. Clinical information can greatly enhance the accuracy of the radiographical diagnosis, i.e. the AIDS patient with an acute airspace process who has chills, fever, and purulent sputum probably has pyogenic rather than a PCP. In the absence of clinical information, radiologists cannot reliably distinguish between pneumonia and other pulmonary processes [10]. Unfortunately, the clinical data and radiographical findings often fail to lead to a definitive diagnosis of pneumonia because there is an extensive number of noninfectious processes associated with febrile pneumonitis i.e. druginduced pulmonary disease, acute eosinophilic pneumonia, bronchiolitis obliterans organizing pneumonia (BOOP), and pulmonary vasculitis that mimic pulmonary infection [11]. Distinction of localized pneumonia from other pulmonary processes cannot be made with certainty on radiological grounds [11, 12]. Localized pulmonary disease of a lobar or segmental distribution can be produced not only by pneumonia but also by pulmonary oedema and haemorrhage. Localized pulmonary oedema produced by acid gastric aspiration may result in an image identical to pneumonia as well as a pulmonary infarction secondary to thromboembolism, which may also produce similar radiographical findings. Diagnosis is equally difficult when pneumonia appears as a diffuse pulmonary abnormality. Pulmonary oedema and the adult respiratory distress syndrome (ARDS) are the most common conditions to be distinguished from bronchopneumonia when a generalized pulmonary abnormality is radiographically demonstrated [13-15].

\section{Conventional chest radiography}

According to American Thoracic Society guidelines, posteroanterior (PA) (and lateral when possible) chest radiography should be obtained whenever pneumonia is suspected in adults [16]. The role of chest radiography has been described either as a screening tool for the detection of new infiltrates or for monitoring response to therapy. Other roles for chest radiography include an enhanced ability to assess the extent of disease, to detect complications (i.e. cavitation, abscess formation, pneumothorax, pleural effusion), and to detect additional or alternative diagnoses and sometimes to guide invasive diagnostic procedures.

In most cases different abnormalities can be identified on chest films. The more common radiographical findings include segmental or lobar consolidations and interstitial lung disease. Other less common radiographical findings include mediastinal lymphadenopathy, pleural effusion, cavitation, and chest wall invasion. Despite that, the nonspecificity of radiographical findings as well as the wide range of potential causes often lead to frustration when evaluating the imaging findings of a patient with suspected pneumonia. Pulmonary infection by PCP, typically seen as a diffuse homogeneous alveolar consolidation, has recently been described, in $5-10 \%$ of cases, with dense consolidation, nodules, miliary opacities, and pleural effusions [16]. Furthermore, equivocal or normal chest radiographs are not uncommon, reported in the range of $10-39 \%$ of patients with PCP infection and in up to $10 \%$ of patients with proven pulmonary disease [17].

\section{Computed tomography}

$\mathrm{CT}$ is a useful adjunct to conventional radiography in selected cases [10,12, 18, 19]. There is a large amount of literature indicating that $\mathrm{CT}$ is a sensitive method capable of imaging the lung with excellent spatial resolution, providing anatomical detail similar to that seen by gross pathological examination. Differences in tissue attenuation and parenchymal changes caused by an acute inflammatory process can be readily seen by CT [18, 19]. Unlike chest radiography, CT provides cross-sectional images and the pattern and distribution of pulmonary processes are therefore, much more readily appreciated than on conventional examinations [17]. 
With the advent of high-resolution CT (HRCT), a whole new lexicon of terminology to describe imaging findings evolved. Recognition of the secondary pulmonary lobule is essential to understand the imaging findings obtained by thin-section CT scans [18]. The findings of airspace disease, airspace (acinar) nodules, ground-glass opacities, consolidation, air bronchograms, and centrilobular or perilobular distribution are seen better by $\mathrm{CT}$ than by conventional radiography $[17,18]$. Airspace nodules represent the size of the acinus $(6-10 \mathrm{~mm})$ and are centrilobular in distribution. They are best appreciated in early disease and best seen at the edge of the pathological process where consolidation is incomplete. Ground-glass opacities are defined as a localized increase in lung attenuation that allows visualization of vascular structures coursing through the affected region. Ground glass is a nonspecific CT finding that may represent either alveolar or interstitial disease [10].

The CT findings of interstitial disease reflect thickening by oedema, neoplasm, inflammation, or fibrosis of the normal interstitial structures $[10,18]$. The most common CT findings are septal thickening, bronchial wall thickening, mosaic perfusion, bronchovascular bundle thickening, interstitial nodules, and honeycombing. These findings, well known from plain film studies, are more easily recognized by CT.

Although CT is not recommended for the initial evaluation of patients with pneumonia, it is a valuable adjunct to conventional radiography in patients with nonrevealing or nondiagnostic imaging findings [16]. Several studies have shown that HRCT can be helpful in the detection, differential diagnosis, and management of immunocompromised patients with pulmonary complications [16-19].

\section{Imaging of pneumonia in specific patient groups}

\section{Community-acquired pneumonia}

CAP is a major healthcare and economic problem because of its high morbidity and mortality rate, and because of its direct and indirect costs of management $[1,3]$. Even in young healthy people, pneumonia has been found to be the major medical cause of lost workdays. Between 485,000 and 1 million patients are hospitalized each year in the USA for treatment of CAP. The cost of inpatient care exceeds outpatient care by a factor of 15-20, and comprise the majority of the estimated $\$ 8.4$ billion spent annually for the care of patients with pneumonia [1, 3, 20, 21].

Hospital admission rates of pneumonia episodes vary $22-51 \%$ of patients with CAP [1]. The mortality is higher in less-developed countries, in the young and the elderly, and varied from $10 \cdot 100,000^{-1}-40 \cdot 100,000^{-1}$ inhabitants in three European countries [20]. Although it is true that the radiographical findings of a pneumonia do not provide a specific aetiological diagnosis, the differential diagnosis may be possible in CAP using radiological pattern recognition. Despite the variability regarding the time between the onset of clinical symptoms and the development of a radiographically visible infiltrate, it is well known that in
CAP the majority of pulmonary infiltrates appear within the time period of $12 \mathrm{~h}$. In these patients, pattern recognition may help to classify groups of potentially underlying organisms favouring a bacterial over a viral aetiology. In CAP, diagnosis and disease management most frequently involve chest radiography and generally do not require the use of other imaging modalities [22].

The spectrum of causative organisms of CAP includes Gram positive bacteria such as Streptococcus pneumoniae (the pneumoccocus), Haemophilus influenzae and Staphylococcus aureus, as well as atypical organisms such as Mycoplasma pneumoniae, Chlamydia pneumoniae, or Legionella pneumophila and viral agents such as the influenza A virus and respiratory syncytial viruses. $S$. pneumoniae is by far the most common cause of complete lobar consolidation [23-25]. Other causative agents that produce complete lobar consolidation include Klebsiella pneumoniae and other Gram negative bacilli, L. pneumophila, $H$. influenzae, and occasionally M. pneumoniae [23-26].

Radiographically, lobar pneumonia appears in the periphery abutting against the pleura and spreads towards the core portions of the lung. Round pneumonia is most often encountered in children than adults and is most often caused by S. pneumoniae (fig. 1) [27]. In children, active tuberculous and fungal infection may also present with nodular or mass-like lesions [27]. Bacterial infections may produce multiple rounded pulmonary nodules or masses, with or without cavitation. This may occur from infection with Nocardia, Aspergillus, Legionella, Q fever, and M. tuberculosis [27-29].

Bronchopneumonia, which is most commonly caused by $S$. aureus and $H$. influenzae, occurs when infectious organisms, deposited on the epithelium of the bronchi, produce acute bronchial inflammation with epithelial ulcerations and fibrinopurulent exudate formation. As a consequence, the inflammatory reaction rapidly spreads through the airway walls and spreads into the contiguous pulmonary lobules.

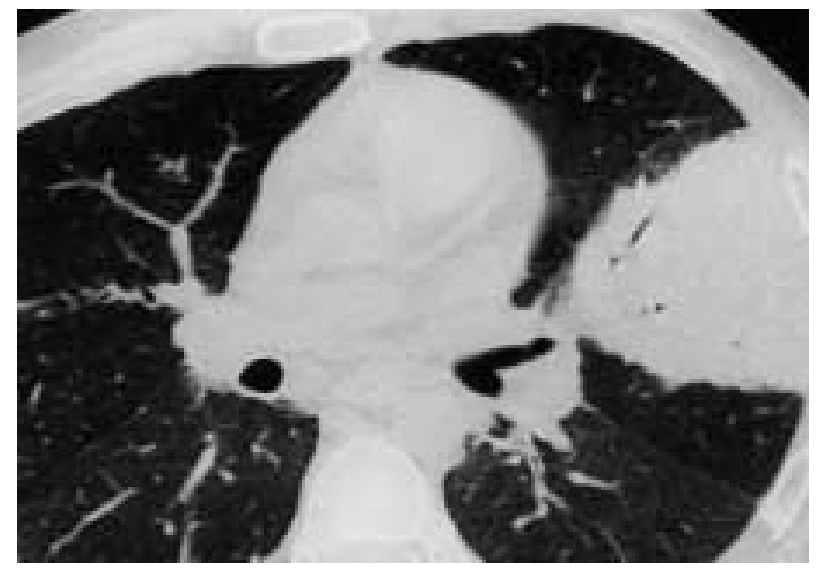

Fig. 1.- Round pneumonia due to Streptococcus pneumoniae in a 53-yr-old male. Computed tomography demonstrates a focal area of homogeneous consolidation in the left upper lobe. Note the presence of air-bronchogram within the consolidation. Sputum culture produced a heavy growth of $S$. pneumoniae. In adults, this form of pneumonia may mimic bronchogenic carcinoma. 


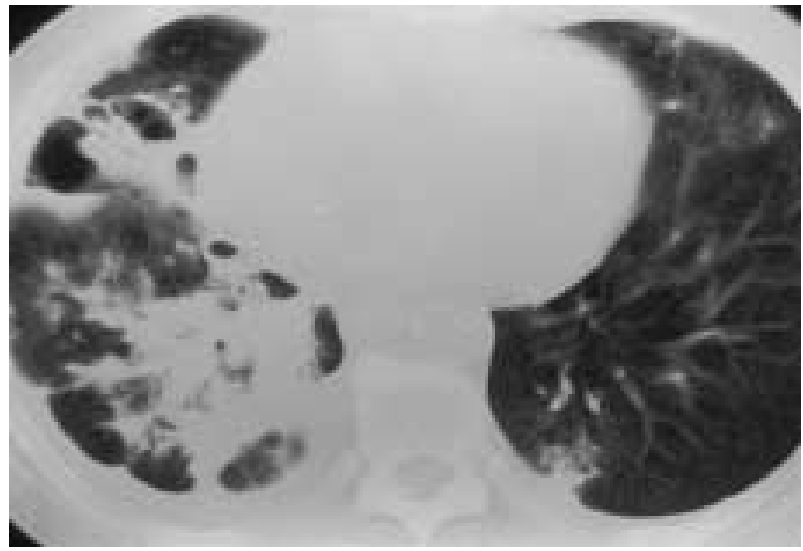

Fig. 2.-Computed tomography scan in a 35-yr-old female demonstrates multiple ill-defined subsegmental opacities in the middle and right lower lobe. Small cavities and moderate right pleural effussion are also appreciated. Note a focus of infection in the left lower lobe. Cultures from a bronchoscopic specimen grew Staphylococcus aureus.

Radiographically, these inflammatory aggregates cause a typical patchy pattern of bronchopneumonia (fig. 2) or a homogeneous segmental consolidation that may also cavitate (figs. 2 and 3 ).

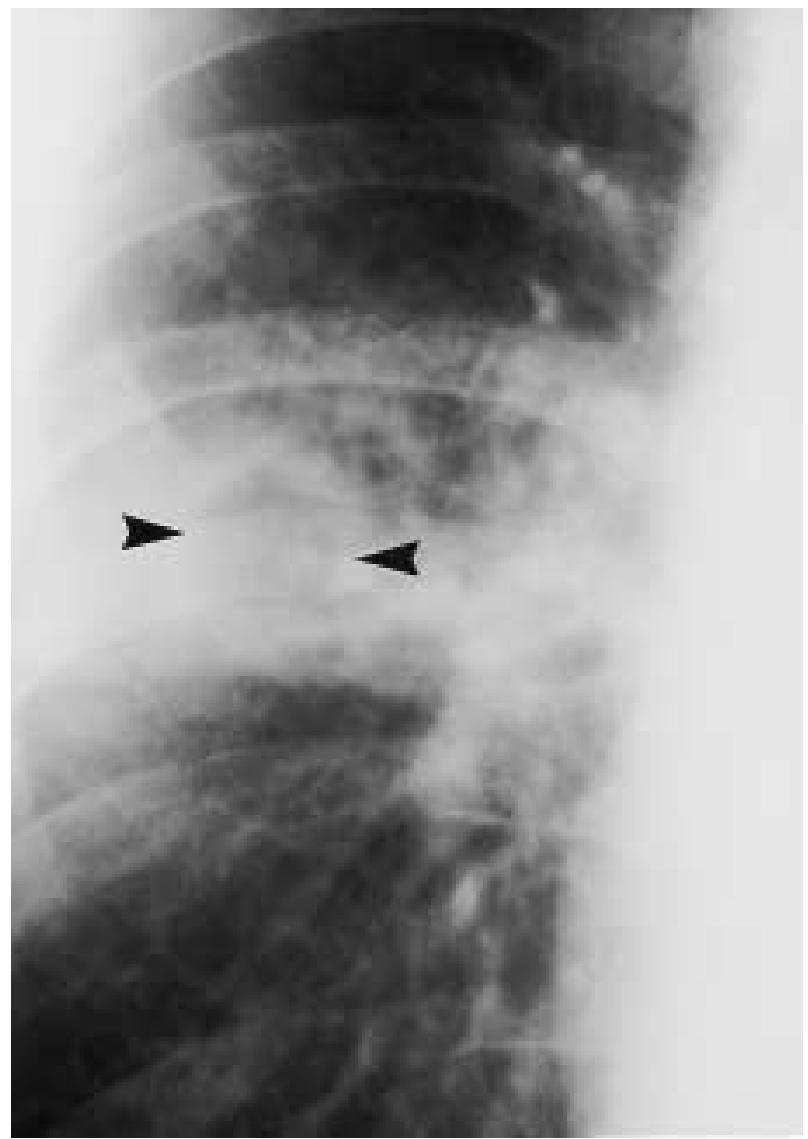

Fig. 3.-Close-up view of a posteroanterior chest radiograph in a 43-yr-old alcoholic male with acute cavitating pneumonia by Staphylococcus aureus. A poorly defined area of airspace consolidation containing a rounded radiolucency (arrowheads) is depicted in the right upper lung.
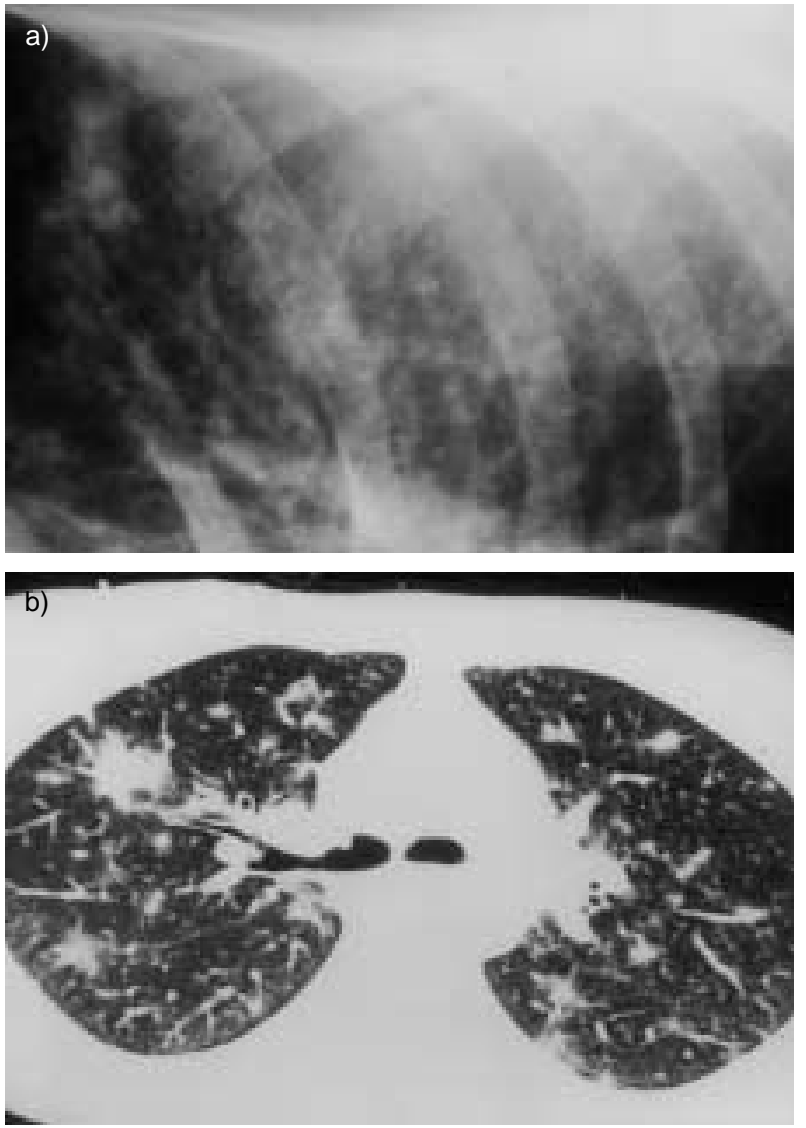

Fig. 4.-Adenovirus pneumonia in a 28-yr-old female. a) Close-up view of a posteroanterior chest radiography demonstrates poorly defined nodular opacities. b) Corresponding high-resolution computed tomography scan shows multiple poorly defined bilateral nodular opacities in a predominantly peribronchial distribution.

Diffuse bilateral interstitial and/or interstitial-alveolar (mixed) infiltrates are most commonly caused by viruses (fig. 4) and M. pneumoniae [30]. Up to $30 \%$ of all pneumonias in the general population may be caused by $M$. pneumoniae [10]. During infection, the initial damage is directed towards the mucosa of the bronchioles and later, the peribronchial tissue and interlobular septa become oedematous and infiltrated with inflammatory cells.

\section{Hospital-acquired (nosocomial) pneumonia}

An NP may be defined as one occurring after admission to the hospital, which was neither present nor in a period of incubation at the time of admission [21]. NP is the leading cause of death from hospitalacquired infections and an important public health problem. It occurs most commonly among intensive care unit (ICU) patients, predominately in individuals requiring mechanical ventilation (fig. 5) [31]. The estimated prevalence of NP within the ICU setting ranges $10-65 \%$, with case fatality rates of $20-55 \%$ in most reported series [26, 31, 32]. In patients with ARDS, as many as $55 \%$ have secondary pneumonia, and this complication may adversely affect survival [26]. 


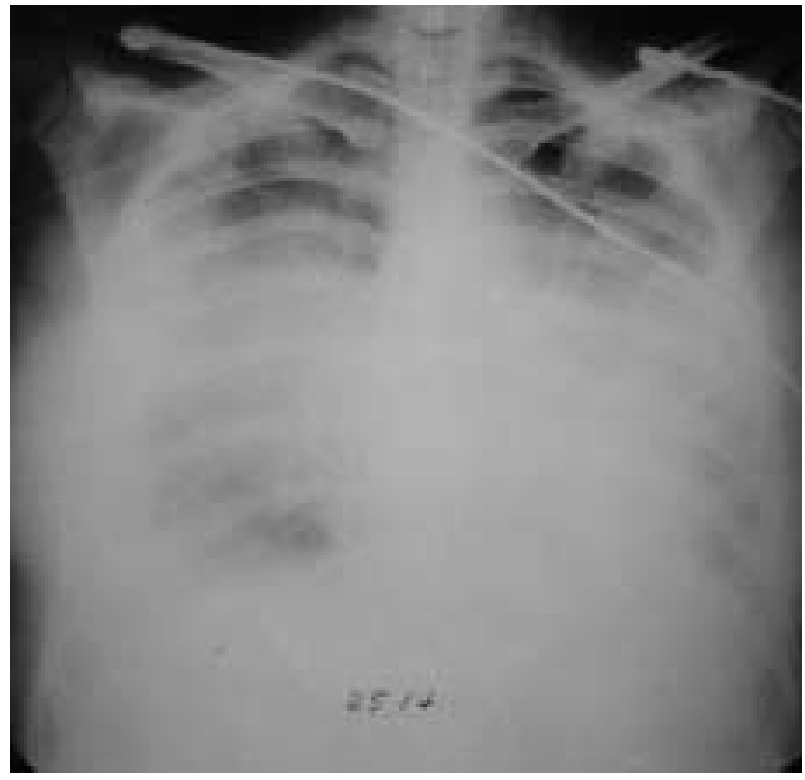

Fig. 5.-Hospital-acquired pneumonia in an intensive care unit patient. Portable anteroposterior supine chest radiography shows bilateral lung consolidation. Protected bronchial brushes revealed Gram positive cocci, gram positive rods, and Gram negative rods on smear. Cultures grew Staphylococcus aureus and Pseudomonas and Serratia organisms.

The diagnosis of NP is difficult, and the criteria used for surveillance have been based on clinical findings of fever, cough, and the development of purulent sputum in combination with a new or progressive infiltrate on the chest radiograph. When pneumonia arises in the hospitalized patient, aerobic Gram negative bacilli, particularly Pseudomonas aeruginosa and Enterobacter spp. and S. aureus, are the major causative organisms [33]. Other common causes of NP are $H$. Influenza, pneumococcus, aspiration with anaerobes, Legionella spp. and viruses in certain hosts. Respiratory syncytial virus, influenza $\mathrm{A}$ and $\mathrm{B}$, and parainfluenza, are responsible for $>70 \%$ of nosocomial viral diseases [33]. The clinical and radiographical clues to the aetiological diagnosis of pneumonia are shown in table 1 .

\section{Immunosuppressed host pneumonia}

Patients with impaired immune function are susceptible to infections by a wide range of organisms $[6,7]$. In the last several decades, AIDS epidemic, advances in the treatment of cancer, organ transplantation, and immunossuppressive therapy has resulted in large numbers of patients who develop abnormalities in their immune system [34-36]. Pneumonia is a major clinical problem for immunosuppressed patients and many of the bacteria causing CAP in the healthy community are also responsible for pneumonia in these risk patients. Mildy impaired host immunity as it occurs in chronic debilitating illness, diabetes mellitus, malnutrition, alcoholism, advanced age, prolonged corticosteroid administration and chronic obstructive lung disease have also been regarded as predisposing factors of pulmonary infections [37].

\section{Acquired immune deficiency syndrome}

In AIDS patients, pulmonary complications may result from a number of infectious and noninfectious

Table 1. - Summary of clinical and radiographical clues to the aetiological diagnosis of pneumonia

\begin{tabular}{|c|c|c|}
\hline Radiographical findings & Clinical circumstance & Organism \\
\hline Segmental consolidation & Community-acquired & S. pneumonia, M. pneumoniae \\
\hline Lobar consolidation & $\begin{array}{l}\text { Community-acquired } \\
\text { Diabetes }\end{array}$ & $\begin{array}{l}\text { S. pneumoniae ( } 2 / 3 \text { of community-acquired pneumonias) } \\
K \text {. pneumoniae } \\
\text { Gram negative bacilli }\end{array}$ \\
\hline Rounded pneumonia & $\begin{array}{l}\text { Community-acquired } \\
\text { Alcoholic }\end{array}$ & S. pneumoniae \\
\hline Bronchopneumonia & Hospital-acquired & $\begin{array}{l}\text { P. aeruginosa, } S \text {. aureus, streptococci, Gram negative bacilli, } \\
\text { anaerobes, } M \text {. pneumoniae, aspiration, } L \text {. pneumophila }\end{array}$ \\
\hline Interstitial pneumonia & Community-acquired (winter) & Virus, M. pneumoniae \\
\hline Cavitation/necrosis & $\begin{array}{l}\text { Aspiration } \\
\text { COPD }\end{array}$ & $\begin{array}{l}\text { S. aureus, Gram negative bacilli, anaerobes, actinomycosis, } \\
\text { M. Tuberculosis } \\
\text { Aspergillus }\end{array}$ \\
\hline Multiple cavitary nodules & Drug addict & S. aureus \\
\hline Pneumatoceles & Postinfluenza & S. aureus \\
\hline Empyema & Complication of pneumonia & $\begin{array}{l}\text { S. pneumoniae } \\
\text { S. aureus } \\
\text { Gram negative bacilli } \\
\text { M. tuberculosis }\end{array}$ \\
\hline Chest wall invasion & Alcoholic & $\begin{array}{l}\text { Actinomycosis } \\
\text { M. tuberculosis } \\
\text { Fungi }\end{array}$ \\
\hline Lymphadenopathy & & $\begin{array}{l}\text { M. pneumoniae } \\
\text { M. tuberculosis }\end{array}$ \\
\hline
\end{tabular}

COPD: chronic obstructive pulmonary disease; S. pneumoniae: Streptococcus pneumoniae; S. aureus: Staphylococcus aureus; M. tuberculosis: Mycobacterium tuberculosis; M. pneumoniae: Mycoplasma pneumoniae; K. pneumoniae: Klebsiella pneumoniae; P. aeruginosa: Pseudomonas aeruginosa; L. pneumophila: Legionella pneumophila. Adapted from [34]. 
causes. Among the infectious pulmonary processes, major causative agents include PCP, $M$. tuberculosis, and MAC complex, in addition to many of the more common Gram positive and negative bacteria $[5,16$, 17]. In the past two decades, a resurgence of tuberculosis (TB) has been seen worldwide, including a number of developing countries in which the disease had been on the decline for many decades. This increase in TB is largely related to cases in AIDS patients [38, 39]. Infection will depend on the patient's immune status and the risk of opportunistic infections will also change over time [39].

Patients who have CD $4+$ cell counts of $>200$ cells $\cdot \mathrm{mm}^{3}$ are predisposed to bronchial infections and bacterial pneumonia, whereas patients with CD4+ cell counts of $<200$ cells $\cdot \mathrm{mm}^{3}$ are predisposed to opportunistic infections such as PCP [8, 39]. Most patients have CD4+ counts in the range of $50-75$ cells $\cdot \mathrm{mm}^{3}$ at the time of diagnosis of their first episode of PCP [8, 17]. Therefore, it is important to interpret the radiological findings in the appropriate clinical setting. By correlating the different radiographic patterns with presenting symptoms and the CD4+ cell count, the radiologist may narrow the differential diagnosis [8]. Abnormal chest radiographs have been reported in up to $90 \%$ of patients showing the typical findings of diffuse bilateral interstitial infiltrates without a pleural effusion (fig. 6). As the disease progresses, alveolar infiltrates may also develop. HRCT is the modality of choice to evaluate those symptomatic patients with an otherwise normal chest radiograph [17].

Bronchial invasive aspergillosis occurs most commonly in the setting of severe neutropenia and in patients with AIDS [40-42]. Clinical manifestations include acute tracheobronchitis, bronchiolitis, and bronchopneumonia. Patients with acute tracheobronchitis usually have normal radiological findings. Aspergillus bronchiolitis is characterized on HRCT by the presence of centrilobular nodules and branching linear or nodular opacities giving an appearance resembling a "tree-in-bud" (fig. 7) [41]. The centrilobular nodules have a patchy distribution in the lung

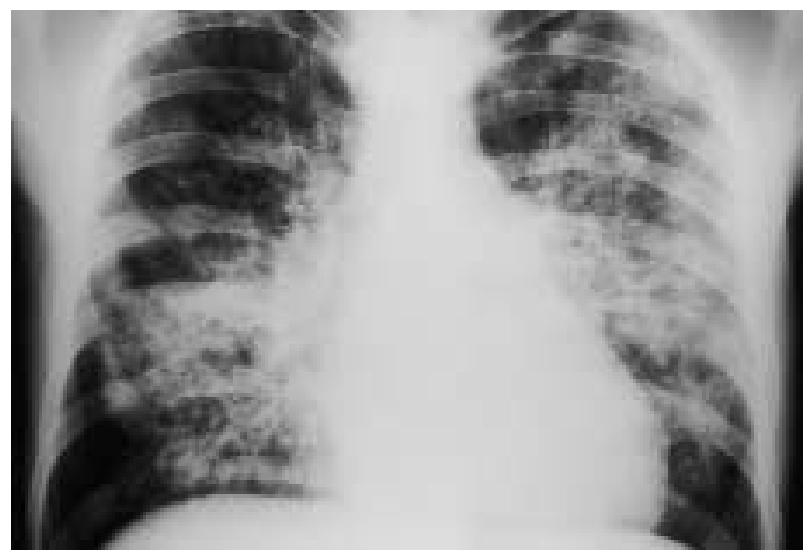

Fig. 6.-Posteroanterior chest radiography in a patient with acquired immune deficiency syndrome and a CD4+ count of 50 cells $\cdot \mathrm{mm}^{3}$. Bilateral asymetric mixed pattern (interstitial and confluent alveolar opacities) are clearly demonstrated. In this clinical setting, radiographical findings are considered highly diagnostic of Pneumocystis carinii pneumonia.

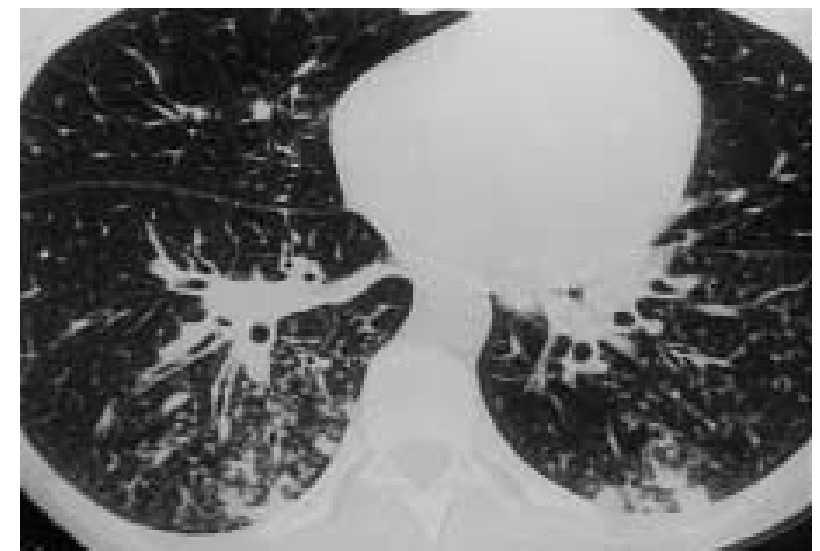

Fig. 7.-A 28-yr-old patient with acute leukaemia presented with fever and a normal chest radiograph. High-resolution computed tomography scan demonstrates thickening of the bronchial and bronchiolar walls and multiple bilateral ill-defined nodular opacities with a "tree-in-bud" appearance. The final diagnosis was Aspergillus bronchiolitis.

and are similar to those seen in a number of different infectious conditions, including endobronchial spread of pulmonary tuberculosis, M. avium-intracellulare, viral and $M$. pneumonia. Aspergillus bronchopneumonia results in predominantly peribronchial areas of consolidation (fig. 8) [41]. Rarely, the consolidation may have a lobar distribution. These radiological manifestations are indistinguishable from those of bronchopneumonia caused by other organisms.

Obstructing bronchopulmonary aspergillosis (OBA) is a descriptive term for the unusual pattern of a noninvasive form of aspergillosis characterized by the massive intraluminal overgrowth of Aspergillus spp., usually Aspergillus fumigatus, in patients with AIDS [42]. Patients may cough up fungal casts of their bronchi and present with severe hypoxaemia. The characteristic CT findings in OBA mimic those of allergic bronchopulmonary aspergillosis (ABPA) consisting of

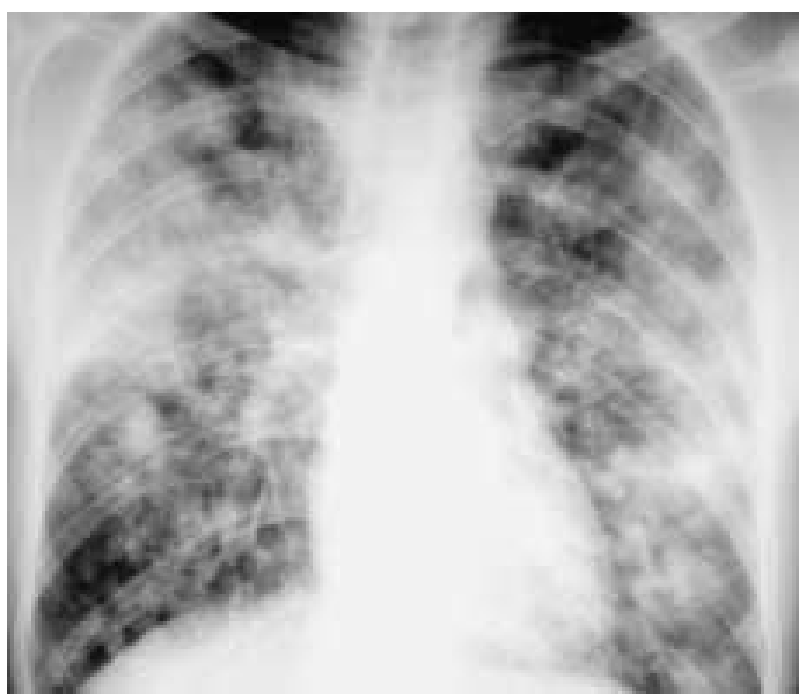

Fig. 8.-Posteroanterior chest radiograph reveals bilateral nonsegmental consolidations in the lingula and in the right upper and lower lobes. Aspergillus fumigatus was recovered from the sputum. 


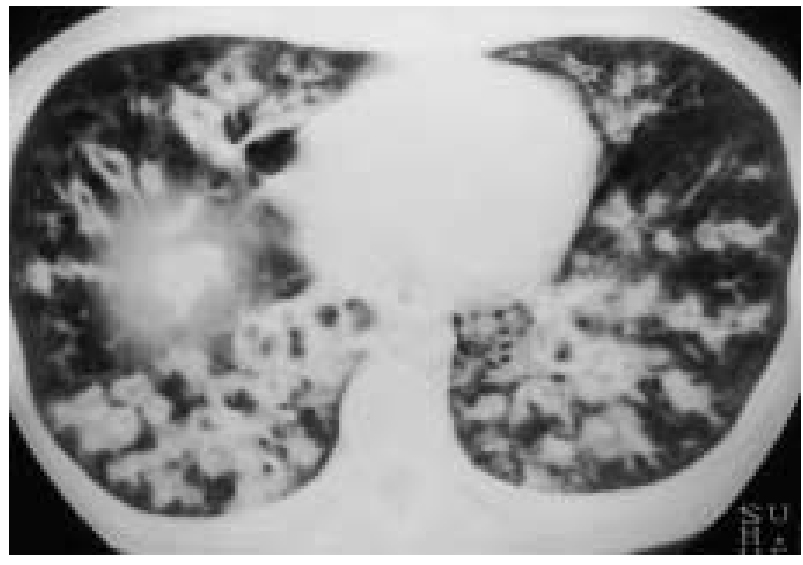

Fig. 9.-Bronchial obstructing aspergillosis in a 24-yr-old male with acquired immune deficiency syndrome. Computed tomography (CT) scan shows bilateral bifurcating tubular shadows caused by impacted mucous material within markedly dilated bronchi. CT findings are similar to those of allergic bronchopulmonary aspergillosis.

bilateral bronchial and bronchiolar dilatations, large mucoid impactions mainly in the lower lobes and diffuse lower lobe consolidation caused by postobstructive atelectasis (fig. 9) [42].

\section{Solid organ transplantation}

Patients undergoing solid organ transplantation present increased susceptibility to infection which varies according to the time interval since transplantation $[35,43,44]$. The post-transplantation timeline can be divided into three periods: 30 days posttransplantation, 30-120 days post-transplantation, and $>120$ days post-transplantation $[35,43,44]$. In the immediate postoperative period opportunistic infections are usually not encountered because there is a delay between the onset of the immunosuppressive therapy and the development of immune system dysfunction. Suppression of the immune system is more severe during the 1-4-month period after organ transplantation. During the first month after heart transplantation, Gram negative bacterial pneumonia are particularly frequent because of prolonged intubation, pulmonary oedema, and the effects of surgery on lung mechanics $[35,36,43,44]$.

Infection rates among lung transplant recipients, occurring in up to $50 \%$ of cases, are several fold higher than among recipients of other solid organs [35]. Both Gram negative bacteria (Enterobacter and Pseudomonas) and Staphylococcus are most common, but they are not lethal as often as viral and fungal infections [35]. CMV infection is the most common viral pathogen encountered in the post-transplantation period. CMV infection typically emerges within the first 3 months after transplantation. Primary infection, the most serious, occurs in $50-100 \%$ of seronegative recipients who receive a graft from a seropositive donor. As many as $40 \%$ of patients undergoing bone marrow transplantation (BMT) develop invasive fungal disease [35]. Aspergillus species commonly colonize the airways of lung transplant recipients but only a minority of patients develop invasive disease. Airway invasive aspergillosis is characterized histologically by the presence of Aspergillus organisms deep to the airway basement membrane $[43,44]$.

\section{Bone marrow transplantation}

BMT is currently the treatment of choice for many haematological malignancies and severe congenital or acquired disorders of the haematopoietic or immune systems [36]. In transplant recipients, pulmonary infections occur in up to $50 \%$ of patients because of direct lung communication with the atmosphere. The new onset of respiratory symptoms, or new infiltrates on chest radiography, should prompt an early and definitive diagnosis.

CMV is the most significant viral infection that occurs in organ and BMT patients. It occurs in $50-70 \%$ of allogeneic BMT recipients [36]. These patients are at a significantly higher risk of pulmonary infection than autologous transplant recipients [36]. CMV infection may be related to primary acquisition or to reactivation of latent infection or re-infection with a different strain in a previously seropositive patient. Approximately one-third of infected patients subsequently develop CMV pneumonia with a median onset time of 50-60 days post-transplantation [36]. CMV infection usually develops 1-4 months after transplantation. The radiographical manifestations of these pneumonias are nonspecific. The radiological findings of CMV infection are variable consisting of lobar consolidation, diffuse and focal parenchymal haziness, and multiple small nodules with associated areas of ground-glass attenuation ("halo") (fig. 10) [45].

Many focal lesions are due to fungal infection, particularly due to Aspergillus species. Opportunistic fungi constitue the second most common group of pathogens with a higher probability of causing infection in allogeneic than in autologous transplant

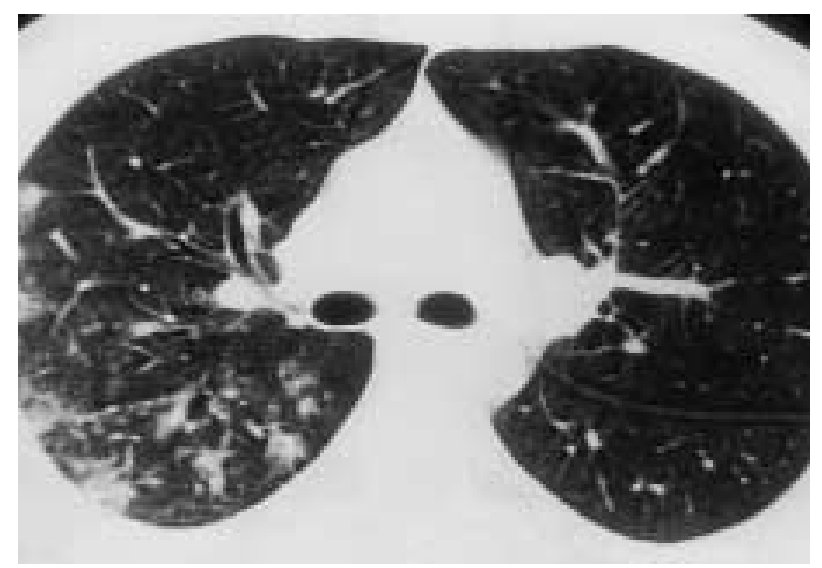

Fig. 10.-Cytomegalovirus pneumonia in a 36-yr-old female after bone marrow transplantation. A high-resolution computed tomography scan demonstrates multiple nodular opacities with irregular margins surrounded by an area of ground-glass attenuation. This halo of ground-glass attenuation is due to the haemorrhagic nature of nodules. 


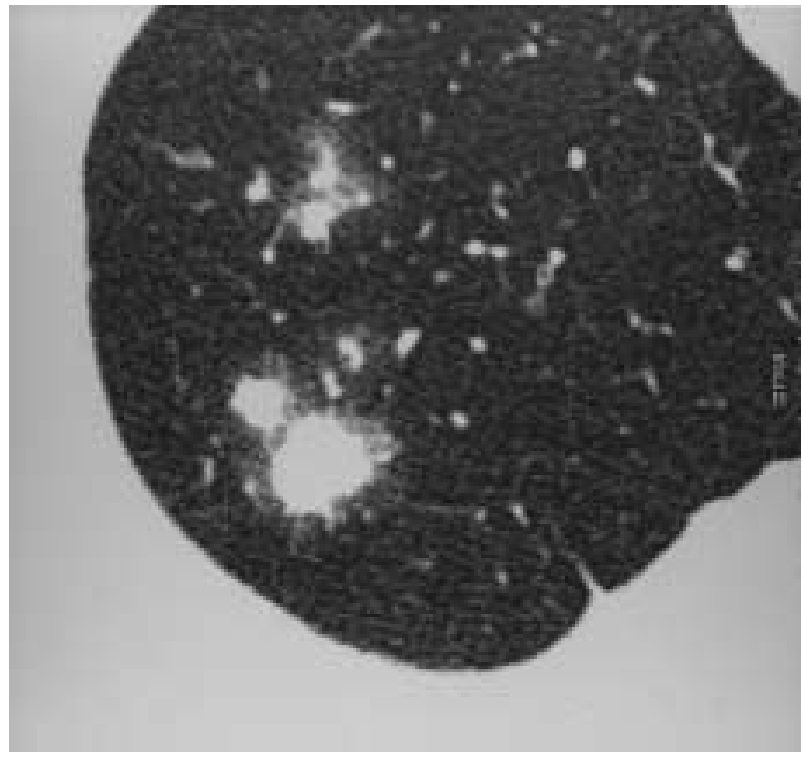

Fig. 11.-Angioinvasive aspergillosis in a 68-yr-old male with severe neutropenia. Magnified view of a computed tomography scan shows a nodule in the left upper lobe surrounded by an halo of ground-glass attenuation (halo sign).

recipients. The most common fungi responsible for acute lung disease in the immunocompromised patient are A. fumigatus, Candida albicans, and Histoplasma capsulatum. Aspergillus is a ubiquitous soil fungus [40]. The histological, clinical and radiological manifestations of pulmonary aspergillosis are determined by the number and virulence of the organisms and by the patient's immune response [40].

Angioinvasive aspergillosis occurs almost exclusively in immunocompromised patients with a severe neutropenia [40-42]. There has been a substantial increase in the number of patients at risk of developing invasive aspergillosis, for many reasons, including the development of new intensive chemotherapy regimens for solid tumours, difficult-to-treat lymphoma, myeloma, and resistant leukaemia as well as an increase in the number of solid organ transplantation and increased use of immunosuppressive regimens for other autoimmune diseases. Angioinvasive aspergillosis is characterized histologically by invasion and occlusion of small to medium pulmonary arteries by fungal hyphae [41]. This leads to the formation of necrotic haemorrhagic nodules or pleural based wedgeshaped haemorrhagic infarcts. The clinical diagnosis is difficult and the mortality is high [40]. The characteristic CT findings consist of nodules surrounded by a halo of ground-glass attenuation (Halo sign) or pleural based wedge-shaped areas of consolidation (fig. 11) [46]. These findings correspond to haemorrhagic infarcts. In severely neutropenic patients the halo sign is highly suggestive of angioinvasive aspergillosis. A similar appearance has been described in a number of other conditions including infection by Mucorales, Candida, herpes simplex and CMV, Wegener's granulomatosis, Kaposi's sarcoma [47] and haemorrhagic metastases.

\section{Mild immunosupression}

Mildy immunocompromised patients with chronic debilitating illness, diabetes mellitus, malnutrition, alcoholism, advanced age, prolonged corticosteroid administration, and chronic obstructive lung disease are prone to develop a distinct form of aspergillus infection called semi-invasive or chronic necrotizing aspergillosis, characterized histologically by the presence of tissue necrosis and granulomatous inflammation similar to that seen in reactivation of TB. [37]. This form of aspergillus infection may be associated with a variety of nonspecific clinical symptoms such as cough, sputum production, and fever for $>6$ months. Haemoptysis has been reported in $15 \%$ of patients with semi-invasive aspergillosis [37].

Radiological manifestations of semi-invasive aspergillosis include unilateral or bilateral segmental areas of consolidation with or without cavitation and/or adjacent pleural thickening, and multiple nodular opacities [37]. The findings progress slowly over months or years. Aspergillus necrotizing bronchitis may be seen on CT as an endobronchial mass, an obstructive pneumonitis and/or collapse, or as a hilar mass. Only a few reports have described the CT findings of aspergillus necrotizing bronchitis involving the central airways; reported abnormalities include circumferential bronchial wall thickening and bronchial obstruction. In clinical practice, the diagnosis of aspergillus necrotizing bronchitis is usually based on the presence of abnormal chest radiography and bronchoscopic biopsy specimen consistent with tissue invasion [37]. The clinical and radiographical clues to the aetiological diagnosis of infection in the immunosuppressed host are shown in table 2 .

\section{Interventional procedures in the patients with pneumonia}

The only definitive way to reach a specific diagnosis is through demonstration of the infected organism, i.e. by examination of stained smears of sputum, pleural fluid or other biological material, by culture of respiratory secretions and blood, or by other interventional procedures. Alternatively, culture of material obtained by transthoracic thin-needle biopsy under fluoroscopy or CT guidance could be a reliable cost-effective means of diagnosis.

However, in most large series of pneumonia a causative organism cannot be identified in $33-45 \%$ of patients, even when extensive diagnostic tests are undertaken. Previously healthly patients who are mildly ill due to pneumonia are managed in an empirical fashion. However, in certain circumstances, the lack of specific organisms requires a more aggressive approach in order to obtain histopathological and cultural identification of the cause of the pulmonary infection.

There has been much debate on the diagnostic accuracy of specimens obtained for culture with various techniques. Material obtained from the sputum or nasopharyngeal secretions have limited diagnostic value because of the presence of normal flora and 
Table 2. - Summary of clinical and radiographical clues to the aetiological diagnosis of infection in the immunosuppressed host

\begin{tabular}{lll}
\hline HRCT findings & Clinical circumstance & Organism \\
\hline Lobar consolidation & Community-acquired & S. pneumoniae \\
& AIDS of 3 of community-acquired \\
pneumonias & Semi-invasive aspergillosis \\
& CD4+ $>200$ cells $\cdot \mathrm{mm}^{3}$ & \\
& Mild immunosuppression & \\
& Diabetes & \\
& Alcoholism & Gram negative bacilli \\
& Solid organ transplantation & Staphylococcus \\
Ground-glass opacity & AIDS & Pneumocystis carinii pneumonia \\
& CD4+ $50-75$ cells $\cdot \mathrm{mm}^{3}$ & CMV \\
Bronchopneumonia & Bone marrow transplant & Bronchial invasive aspergillosis \\
Interstitial pneumonia & Neutropenia & CMV \\
& Bone marrow transplant & Pneumocystis carinii pneumonia \\
Multiple small nodules & AIDS & CMV \\
& Bone marrow transplant & Cryptococcosis \\
& AIDS & Varicella \\
Multiple cavitary nodules & & Herpes \\
"Halo sign" & S. aureus \\
"Tree-in-bud" & Drug addict & Angioinvasive aspergillosis \\
& Neutropenia & Bronchial infection \\
& AIDS & Endobronchial spread of \\
Lymphadenopathy & CD4+ $>200$ cells $\cdot \mathrm{mm}^{3}$ & tuberculosis \\
& Transplantation & Aspergillosis \\
& AIDS & M. tuberculosis
\end{tabular}

AIDS: acquired immune deficiency syndrome; COPD: chronic obstructive pulmonary disease; S. pneumoniae:Streptococcus pneumoniae; CMV: cytomegalovirus; S. aureus: Staphylococcus aureus; M. tuberculosis: Mycobacterium tuberculosis; HRCT: high-resolution computed tomography.

variable results obtained for the detection of anaerobic infection [48].

\section{Flexible fibreoptic bronchoscopy with lung biopsy}

Fibreoptic bronchoscopy with bronchoalveolar lavage utilizing a protected brush is a well-established technique in the diagnosis of pulmonary infection. Although this technique may play an important role in the diagnosis of pulmonary infection, the yield of bronchoalveolar lavage is variable and sometimes the diagnosis of a pulmonary infection cannot be established $[49,50]$. This method has proved particularly useful in the diagnosis of Pneumocystis pneumonia in AIDS patients, providing an aetiological diagnosis in $\sim 95 \%$ of cases.

In the special setting of a serious pulmonary process and lack of definable cause with noninvasive methods, fibreoptic bronchoscopy in conjunction with transbronchial lung biopsy is indicated (fig. 12).

\section{Transthoracic-needle aspiration}

Despite the fact that reported results in the diagnosis of pulmonary infection are variable $(11.7-73 \%)$, percutaneous fine-needle aspiration is an alternative method used to identify causative pathogens in selected patients with pneumonia [51-55]. Transthoracicneedle aspiration should be considered for patients who have not responded to initial therapy, who may have nosocomial superinfection, who are immunocompromised, or in whom TB is suspected but has not been confirmed by examination of the sputum or gastric lavage. It is not clear whether use of transthoracicneedle aspiration results in a reduction in mortality and morbidity in a cost-effective fashion, compared to a less invasive approach [48]. The specificity and positive predictive value of a positive culture have been reported to be as high as $100 \%$, whereas the sensitivity and negative predictive value are $61 \%$ and $34 \%$ [56].

\section{Strategies for optimal imaging evaluation}

Chest radiography should be carried out in all patients suspected of having pulmonary infection to confirm or exclude the presence of pulmonary abnormalities. Although radiographical abnormalities can never establish aetiological sources, they can be extremely helpful in narrowing the differential diagnosis and providing guidance for subsequent diagnostic studies.

In patients with CAP, diagnosis and disease management most frequently rely on conventional chest films and usually do not require the use of further diagnostic procedures. In the community setting, $>90 \%$ of patients who develop a segmental or lobar consolidation have either pneumococcal pneumonia or an atypical pneumonia caused by Mycoplasma or a virus. 

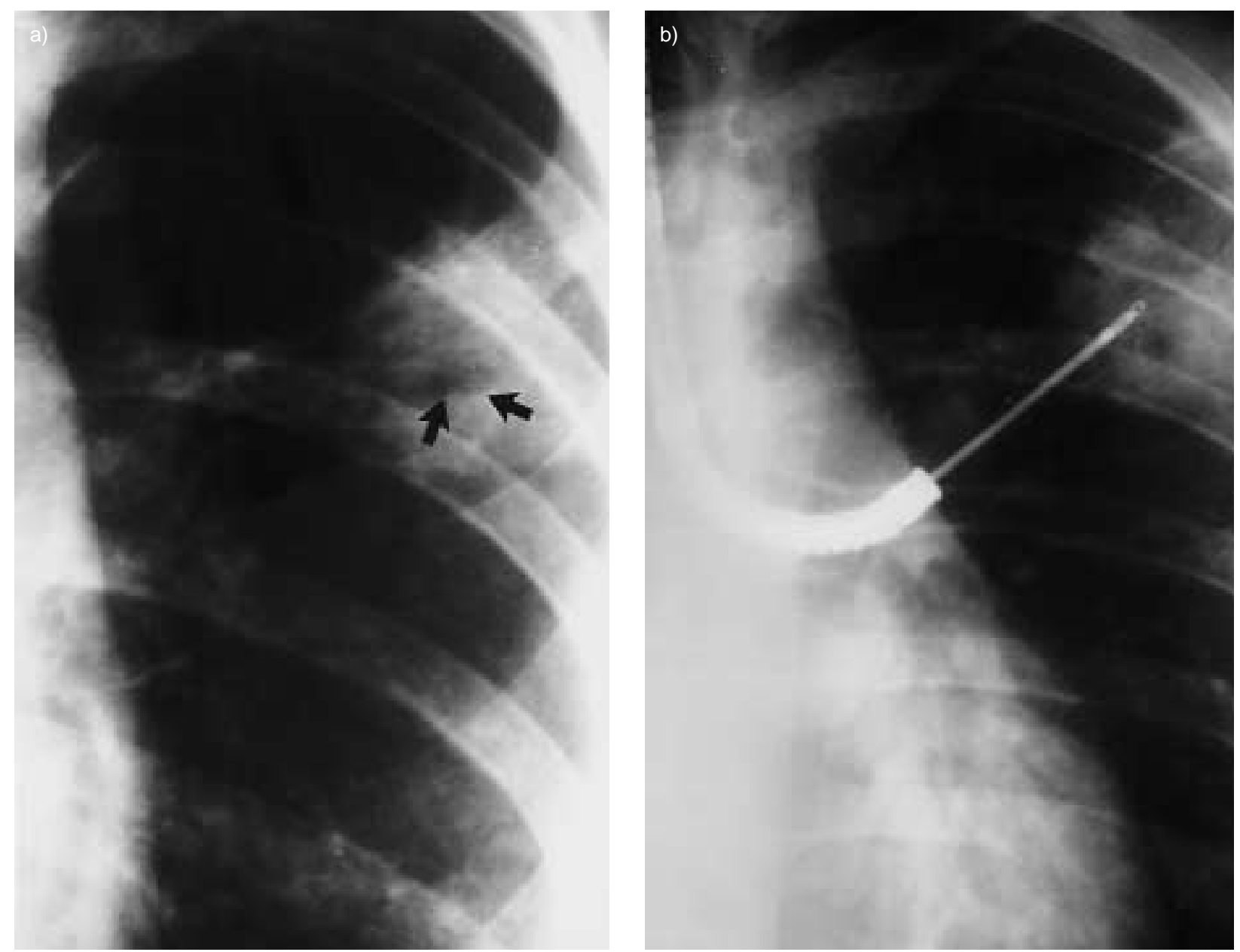

Fig. 12.-a) Close-up view of a posteroanterior chest radiography shows a rounded cavitary consolidation in the left upper lobe. b) Material for culture was obtained through fibreoptic bronchoscopy. Cultures grew Mycobacterium tuberculosis.

In NP infection, patchy bronchopneumonia is the most common finding and is most likely caused by one of the Gram negative organisms, particularly Pseudomonas or Klebsiella. In this particular setting, aspiration pneumonia is always an alternative diagnosis and should be suspected if pneumonia is present bilaterally in the dependent or posterior portions of the lungs [57]. In the ICU patients, there are few studies regarding the accuracy and efficacy of conventional chest radiography. The overall incidence of abnormalities found on chest films in the medical ICU has been reported to be as high as $57 \%$ in pulmonary and unstable cardiac patients [57]. Similar results were obtained in a study of patients in the medical ICU; $43 \%$ of routine chest radiographs showed unexpected findings which influenced therapy [58]. Future studies on management and outcome efficay as well as overall cost are necessary to evaluate the role of the routine chest radiograph in ICU patients. Limiting the need for conventional chest radiography in the follow-up of pulmonary infections may also reduce health costs. CT and invasive diagnostic procedures should be reserved only for complicated cases.

Conversely, management of immunocompromised patients is challenging and difficult because of the diversity of causative organisms. In this group of patients, thin-section CT and invasive procedures are more often required. HRCT can be useful in patients who have respiratory symptoms but normal results on chest films, providing further additional findings not clearly delineated by the standard chest radiograph, depicting concurrent parenchymal or pleural disease, and guiding diagnostic manoeuvres. In addition, HRCT is helpful in differentiating infectious from noninfectious acute parenchymal lung disease despite its limited value in making a specific diagnosis [19].

Diagnostic information may also be obtained by means of bronchoalveolar lavage and transbronchialneedle aspiration. Under these circumstances, CT is extremely useful serving as a "road map" to direct fibreoptic bronchoscopy toward the lesion. Algorithm for evaluation of patients suspected of having pulmonary infection is shown in figure 13.

In conclusion, the radiologist plays an important role in the diagnosis and management of patients with suspected pneumonia. Conventional chest radiography remains the first imaging procedure in the imaging work-up patients. Although computed tomography is not recommended for the initial evaluation, it is frequently appropriate in those cases with normal, 
Suspected pulmonary infection
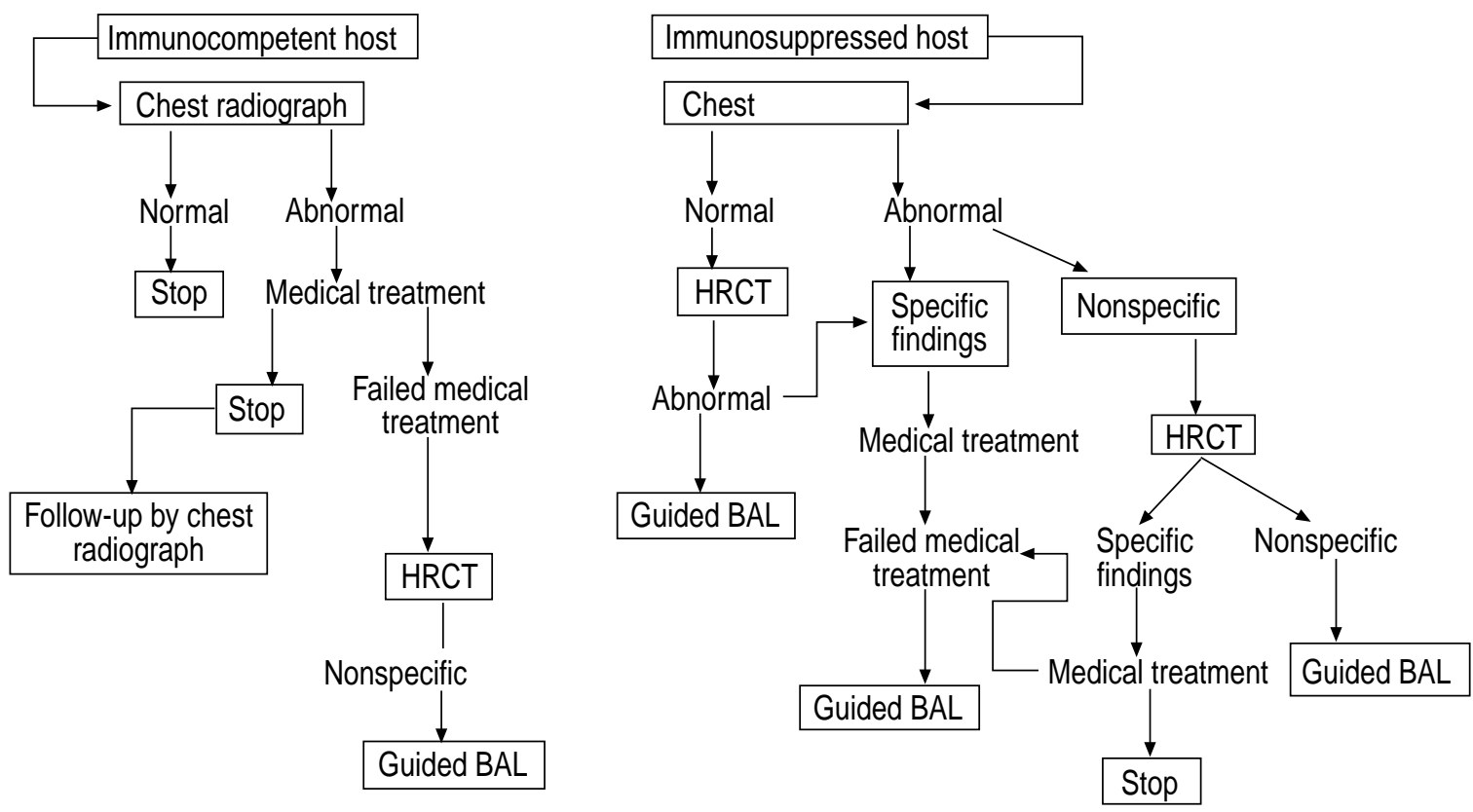

Fig. 13.-Algorithm for evaluation of patients suspected of having pulmonary infection. HRCT: high-resolution computed tomography; BAL: bronchoalveolar lavage fluid.

equivocal, or nonspecific radiographical findings. High-resolution computed tomography is helpful in the differential diagnosis of infectious from noninfectious acute parenchymal lung disease but does not provide the aetiological agent. Percutaneous needle aspiration using fluoroscopy and/or computed tomography is a safe and useful diagnostic method of obtaining specimens in immunocompromised patients with pulmonary infection, although its impact on morbidity and mortality remains to be proved.

\section{References}

1. Niederman MS, McCombs JS, Unger AN, Kumar A, Popovian R. The cost of treating community acquired pneumonia. Clin Ther 1998; 20: 820-837.

2. Vincent JL, Bihari DJ, Suter PM, et al. The prevalence of nosocomial infection in intensive care units in Europe. JAMA 1995; 274: 634-644.

3. Garibaldi RA. Epidemiology of community-acquired respiratory tract infections in adults: incidence, etiology, and impact. Am J Med 1985; 78: Suppl. 6B, 32-37.

4. Lung disease data 1994. New York, American Lung Association, 1994; 37-42.

5. Moe AA, Hardy WD. Pneumocystis carinii infection in the HIV-seropositive patient. Infect Dis Clin North Am 1994; 8: 331-364.

6. Murray JF, Mills J. Pulmonary infectious complications of human immunodeficiency virus infection. Am Rev Respir Dis 1990; 141: 1356-1372.

7. Lyon R, Haque AK, Asmuth DM, Woods GL. Changing patterns of infections in patients with AIDS: A study of 279 autopsies of prison inmates and nonincarcerated patients at a university hospital in eastern Texas, 1984-1993. Clin Infect Dis 1996; 23: 241-247.

8. Shah RM, Kaji AV, Ostrum BJ, Friedman AC. Interpretation of chest radiographs in AIDS patients: usefulness of CD4 lymphocyte counts. Radiographics 1997; 17: 47-58.

9. Hanson DL, Chu SY, Farizo KM, Ward JW. Distribution of CD4 lymphocytes at diagnosis of acquired immunodeficiency syndrome-defining and other human immunodeficiency virus-related illnesses. Arch Intern Med 1995; 155: 1537-1542.

10. Primack SL, Müller NL. HRCT in acute diffuse lung disease in the immunocompromised patient. Radiol Clin North Am 1994; 32: 731-744.

11. Boiselle PM, Tocino I, Hooley RJ, et al. Chest radiograph interpretation pf Pneumocystis carinii pneumonia, bacterial pneumonia, and pulmonary tuberculosis in HIV-positive patients: accuracy, distinguishing features, and mimics. $J$ Thorac Imaging 1997; 12: 47-53.

12. Janzen DL, Padley SPG, Adler BD, Müller NL. Acute pulmonary complications in immunocompromised non-AIDS patients: Comparison of diagnostic accuracy of CT and chest radiography. Clin Radiol 1993; 47: $159-165$.

13. Chastre J, Trouillet JL, Vuagnat A, et al. Nosocomial pneumonia in patients with acute respiratory distress syndrome. Am J Respir Crit Care Med 1998; 157: $1165-1172$.

14. Seidenfeld JJ, Pohl DF, Bell RD, Harris GD, Johnson WG Jr. Incidence, site and outcome of infections in patients with adult respiratory distress syndrome. $\mathrm{Am}$ Rev Respir Dis 1986; 134: 12-16.

15. Niederman MS, Fein AM. Sepsis syndrome, the adult respiratory distress syndrome and nosocomial pneumonia: a common clinical sequence. Clin Chest Med 1990; 11: 633-656. 
16. Boiselle PM, Crans CA Jr, Kaplan MA. The changing face of Pneumocystis carinii pneumonia in AIDS patients. AJR 1999; 172: 1301-1309.

17. Gruden JF, Huang L, Turner J, et al. High-resolution CT in the evaluation of clinically suspected Pneumocystis carinii pneumonia in AIDS patients with normal, equivocal, or nonspecific radiographic findings. AJR 1997; 169: 967-975.

18. Brown MJ, Miller RR, Müller NL. Acute lung disease in the immunocompromised host: CT and pathologic findings. Radiology 1994; 190: 247-254.

19. Tomiyama N, Müller NL, Johkoh T, et al. Acute parenchymal lung disease in immunocompetent patients: diagnostic accuracy of high-resolution CT. AJR 2000; 174: 1745-1750.

20. Jokinen C, Heiskanen L, Juvonen H, et al. Incidence of community-acquired pneumonia in the population of four municipalities in eastern Finland. $A m \mathrm{~J}$ Epidemiol 1993; 137: 977-988.

21. Finch RG, Woodhead MA. Practical considerations and guidelines for the management of communityacquired pneumonia. Drugs 1998; 55: 31-45.

22. Tanaka N, Matsumoto T, Kuramitsu T, et al. High resolution CT findings in community-acquired pneumonia. J Comput Assist Tomogr 1996; 20: 600-608.

23. Kantor HG. The many radiologic facies of pneumoccocal pneumonia. AJR 1981; 137: 1213-1220.

24. Dietrich PA, Jonhson RD, Fairbank JT, Walke JS. The chest radiograph in Legionnarie's disease. Radiology 1978; 127: 577-582.

25. Cameron DC, Borthwick RN, Philp T. The radiographic patterns of acute Mycoplasma pneumonitis. Clin Radiol 1977; 28: 173-180.

26. American Thoracic Society. Hostpital-acquired pneumonia in adults: diagnosis, assessment of severity, initial antimicrobial thereapy, and preventive strategies. Am J Respir Crit Care Med 1996; 153: 17111725.

27. Eggli KD, Newman B. Nodules, masses, and pseudomasses in the pediatric lung. Radiol Clin North Am 1993; 31: 651-666.

28. Quagliano PV, Das Narla L. Legionella pneumonia causing multiple cavitating pulmonary nodules in a 7-month-old infant. AJR 1993; 161: 367-368.

29. Kwong JS, Müller NL, Godwin JD, Aberle D, Grymaloski MR. Thoracic actinomycosis: CT findings in eight patients. Radiology 1992; 183: 189-192.

30. Ettinger NA. Invasive diagnostic approaches to pulmonary infiltrates. Semin Respir Infect 1993; 8: $168-176$

31. Ibrahim EH, Ward S, Sherman G, Kollef MH. A comparative analysis of patients with early-onset vs. late-onset nosocomial pneumonia in the ICU setting. Chest 2000; 117: 1434-1442.

32. Kollef MH. The prevention of ventilator-associated pneumonia. N Engl J Med 1999; 340: 627-634.

33. Taylor GD, Buchanan-Chell M, Kirkland T, McKenzie M, Wiens R. Bacteremic nosocomial pneumonia: a 7 years experience in one institution. Chest 1995; 108 : 786-788.

34. Woodring JH. Pulmonary bacterial and viral inspections. In: Freundlinch IM, Bragg DG, eds. A Radiologic Approach to Diseases of the Chest. Baltimore, Williams \& Wilkins, 1997; p. 436.

35. Fishman JA, Rubin RH. Infection in organ transplant recipients. N Engl J Med 1998; 338: 1741-1751.

36. Cunningham I. Pulmonary infections after bone marrow transplant. Sem Respir Infect 1992; 7: 132138.

37. Franquet $\mathrm{T}$, Müller NL, Giménez A, Domingo $\mathrm{P}$, Plaza V, Bordes R. Semiinvasive pulmonary aspergillosis in chronic obstructive pulmonary disease: radiologic and pathologic findings in nine patients. AJR 2000; 174: 51-56.

38. Chin DP, Hopewell PC. Mycobacterial complications of HIV infection. Clin Chest Med 1996; 17: 697-711.

39. Haramati LB, Jennyavital ER, Alterman DD. Effect of HIV status on chest radiographic and CT findings in patients with tuberculosis. Clin Radiol 1997; 52: 3135.

40. Denning DW, Follansbee SE, Scolaro M, Norris S, Edelstein H, Stevens DA. Pulmonary aspergillosis in acquired immunodeficiency syndrome. $N$ Engl $\mathrm{J} \mathrm{Med}$ 1991; 324: 654-662.

41. Aquino SL, Kee ST, Warnock ML, Gamsu G. Pulmonary aspergillosis: imaging findings with pathologic correlation. AJR 1994; 163: 811-815.

42. Miller WT Jr, Sais GJ, Frank I, Gefter WB, Aronchick JM, Miller WT. Pulmonary aspergillosis in patients with AIDS. Chest 1994; 105: 37-44.

43. Maurer JR, Tullis E, Grossman RF, Vellend H, Winton TL, Patterson GA. Infectious complications following isolated lung transplantation. Chest 1992; 101: 1056-1059.

44. Herman SJ. Radiologic assessment after lung transplantation. Radiol Clin North Am 1994; 32: 663678.

45. McGuiness G, Scholes JV, Garay SM, Leitman BS, McCauley DI, Naidich DP. Cytomegalovirus pneumonitis: spectrum of parenchymal CT findings with pathologic correlation in 21 AIDS patients. Radiology 1994; 192: 451-459.

46. Kuhlman JE, Fishman EK, Siegelman SS. Invasive pulmonary aspergillosis in acute leukemia: characteristic findings on CT, the CT halo sign, and the role of CT in early diagnosis. Radiology 1985; 157: 611-614.

47. Primack SL, Hartman TE, Lee KS, Müller NL. Pulmonary nodules and the CT halo sign. Radiology 1994; 190: 513-515.

48. Sanchez-Nieto JM, Torres A, García-Cordoba F, et al. Impact of invasive and noninvasive quantitative culture sampling on outcome of ventilator-associated pneumonia. Am J Respir Crit Care Med 1998; 157: 371-376.

49. Jolis R, Castella J, Puzo C, Coll P, Abeledo C. Diagnostic value of protected BAL in diagnosing pulmonary infections in inmmunocompromised patients. Chest 1996; 109: 601-607.

50. Castellino RA, Blank N. Etiologic diagnosis of pulmonary infection in immunocompromised patients by fluoroscopically guided percutaneous needle aspiration. Radiology 1979; 132: 563-567.

51. Johnston WW. Percutaneous fine needle aspiration biopsy of the lung: a study of 1015 patients. Acta Cytol 1984; 28: 218-224.

52. Pelmutt LM, Johnston WW, Dunnick NR. Percutaneous thransthoracic needle aspiration: a review. AJR 1989; 152: 451-455.

53. White DA. Pulmonary infection in the immunocompromised patient. Sem Thorac Cardiovasc Surg 1995; 7: 78-87.

54. Haverkos HW, Downling JN, Pasculle AW, Myelowitz RL, Lerberg DB, Hakala TR. Diagnosis of pneumonitis 
in immunocompromised patients by open lung biopsy.

Cancer 1983; 52: 1093-1097.

55. Hwang SS, Kim HK, Park SH, Jung JI, Jang HS. The value of CT-guided percutaneous needle aspiration in inmmunocompromised patients with suspected pulmonary infection. AJR 2000; 175: 235-238.

56. Dorca J, Manresa F, Esteban L, et al. Efficacy, safety, and therapeutic ultrathin needle in nonventilated nosocomial pneumonia. Am J Respir Crit Care Med 1995; 151: 1491-1496.

57. Strain DS, Kinasewitz GT, Vereen LE, George RB. Value of routine daily chest $\mathrm{x}$-rays in the medical intensive care unit. Crit Care Med 1985; 13: 534-536.

58. Greenbaum DM, Marshall KE. The value of routine daily chest x-ray in intubated patients in the medical intensive care unit. Crit Care Med 1982; 10: 29-30. 